\title{
Telephysiotherapy as a Mode of Enhancing Motor Skills of Cerebral Palsy Children in School Settings: A Review
}

\author{
Pardeep K. Pahwa and Suresh Mani
}

\begin{abstract}
Aim: The aim of this review article was to the discuss about role of telephysiotherapy for cerebral palsy children in school settings by searching all relevant data. Method: A total of 46 journal articles were selected first. With the different combinations of Key terms, articles were screened on relevance based on the inclusion and exclusion criteria which resulted in 11 articles for this review.Results: Various researchers reported the effect of telephysiotherapy on gross and fine motor skills along with ADLs in cerebral palsy children in school settings. Conclusion: Review of articles revealed that telephysiotherapy for Cerebral Palsy children is effective mode of treatment in enhancing motor skills along in educational settings.
\end{abstract}

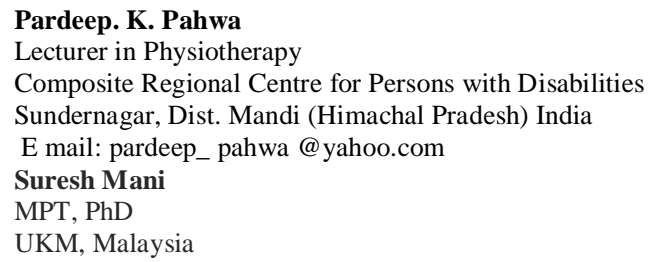

\author{
Key Words: Cerebral Palsy, \\ Telerehabilitation, Virtual Reality, \\ Physical, Technology
}

DOI: $10.18376 / j e s p / 2018 / v 14 / \mathrm{i} 2 / 111302$

\section{Introduction}

The Telerehabilitation is an emerging field and its scope is very vast in medical and other related fields, since it faces challenges related to both medical and community care settings. In the last few years, research has demonstrated the potential for improving telerehabilitation processes based not only on mobile technologies and the internet in general, but also on virtual reality. The aim of telerehabilitation is to provide rehabilitation services at a distance to help people to regain their psycho-physical functions through the use of new technologies. Medical telerehabilitation is more focused on curative medicine and involves intensively trained clinicians and different health professionals particularly physiotherapists (Thais et.al, 2014). Cerebral palsy is a neurodevelopment disorder characterized by movement and posture abnormalities. Incidence of $\mathrm{CP}$ in countries of the western world is approximately 2-3 per 1,000 births. Children with CP usually show signs of muscle weakness, sensory deficits as well as spasticity and accompanied by loss of functionality and dependence on others for many daily activities. Children with cerebral palsy, as well as other motor disabled individuals, have different motor abilities and thus the capabilities of learning a new skill (Josip et. al., 2016). A cure for CP, which means repair of the underlying brain damage, is not currently available; therefore, the management of children with CP usually focuses on maintaining and improving quality of life and function and preventing secondary complications. Patients with $\mathrm{CP}$ are at a high risk of developing musculoskeletal problems that are mainly related to physical growth, abnormal muscle tone, a weakness, lack of mobility, poor balance and loss of selective motor control. (Cristinia et al., 2016). Telerehabilitation techniques mimic virtual reality and rehabilitation for neurological conditions by using robotics and gaming techniques. Telerehabilitation allows for treatment of the acute phase of diseases by substituting the traditional face-to-face approach in the patient rehabilitation interaction (Alessandro et al., 2017). Families are choosing educational programming for their students that provide learning opportunities using 
Internet-based methods. Despite the ability to access teacher support on-line, all students with special needs must still have access to appropriate special education services, including related services of speech therapy, occupational therapy and physical therapy (Criss 2013). The aim of telerehabilitation is to provide rehabilitation services at a distance to help people to regain their psycho-physical functions through the use of new technologies. The scope of telerehabilitation is vast, since it faces challenges related to both medical and community care settings. In the last few years, research has demonstrated the potential for improving telerehabilitation processes based not only on mobile technologies and the Internet in general, but also on virtual reality (Gilberto and Velta 2016). Telerehabilitation is a delivery model that uses telecommunications technology to provide therapeutic services at a distance. Telerehabilitation is a feasible service delivery model that has the potential to improve access and reduce the costs associated with delivery of early intervention services in rural communities (Jana 2011). Telehealth technology is effective in delivering paediatric acute care to children in schools. Paediatric providers, nurses, parents, and children reported primary care school-based telehealth as an acceptable alternative to traditional health care delivery systems (Young and Ireson 2003).

\section{Materials and Methods}

This is a review article collected from period of 2010 to 2017. The collected sources are from databases like Pub Med, Science direct and Pedro along with books and thesis. The search was based on the following MeSH terms as "Cerebral Palsy", "Video Games" and "Tele Rehabilitation". When no MeSH - term was available, the following key-terms were used like "virtual", "virtual reality", "virtual environment". When combining MeSH and Key terms, this resulted in a total of 46 articles, of which 34 articles were founded in Pub Med and 12 in Pedro. With the different combinations of Mesh and Key terms, a lot of double articles were found. In PubMed there were 12, in Pedro there were 10 double articles. The total articles after exclusion of the double articles were 22. These 22 articles were screened on relevance based on the inclusion and exclusion criteria which resulted in 13 articles but only 11 articles were selected for this review. Because of poor evidence about telerehabilitation physiotherapy in children with $\mathrm{CP}$, case studies, pilot studies were also included. Selection criteria were made by use of PICO.

\begin{tabular}{|l|l|}
\hline Population & Children with CP \\
\hline Intervention & Telephysiotherapy rehabilitation \\
\hline Comparator & Motor skills \\
\hline Outcome & Results of motor learning \\
\hline Study design & Clinical trials, case reports, pilot study and review \\
\hline
\end{tabular}

During the screening of the articles, the following inclusion and exclusion criteria were used:

Inclusion Criteria

1) Selected articles in English language.

2) Population: Cerebral Palsy children.

3) Intervention: Use of telerephysiotherapy as a tool for rehabilitation.

4) Outcome: Effect of telephysiotherapy intervention on motor skills.

Exclusion Criteria

1) All other disabled children like developmentally delayed, spina bifida.

2) All other forms of intervention or rehabilitation measures used for Cerebral Palsy children. 


\section{Exclusion procedure by literature review}

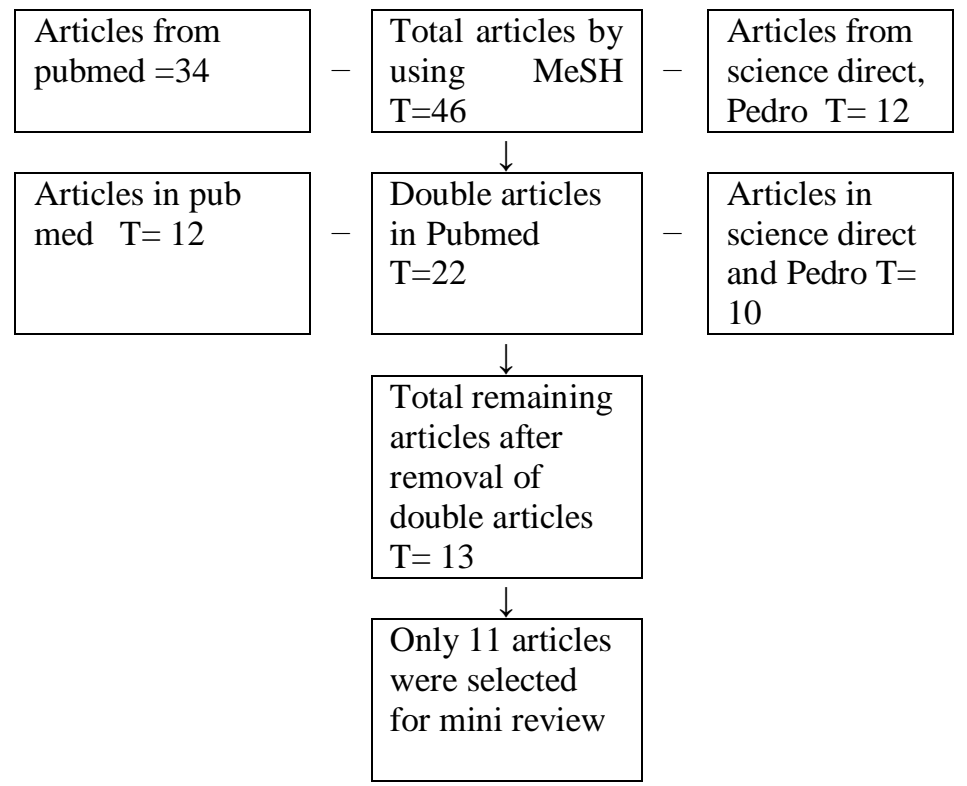

\section{Results}

After data extraction based on the exclusion criteria, the search resulted in

\begin{tabular}{|c|c|c|c|c|c|c|}
\hline S.No. & Author & $\begin{array}{l}\text { Article } \\
\text { type }\end{array}$ & $\begin{array}{l}\text { Population, } \\
\text { time duration }\end{array}$ & Intervention & $\begin{array}{l}\text { Measurement } \\
\text { outcome }\end{array}$ & $\begin{array}{l}\text { Tool } \\
\text { of } \\
\text { measurement }\end{array}$ \\
\hline 1 & $\begin{array}{l}\text { Josip } \\
\text { Glavic et } \\
\text { al }\end{array}$ & $\begin{array}{l}\text { Case study, } \\
\text { Age }=18 \\
\text { years. }\end{array}$ & $\begin{array}{l}\text { Spastic cerebral } \\
\text { palsy children } \\
\text { Training } \\
\text { frequency = } \\
5 \text { times per } \\
\text { week for } 12 \\
\text { weeks. } \\
\text { Session=40 min. }\end{array}$ & $\begin{array}{l}\text { Robotic } \\
\text { assisted } \\
\text { training using } \\
\text { armeo spring } \\
\text { system }\end{array}$ & $\begin{array}{l}\text { Improving } \\
\text { upper limb } \\
\text { functions }\end{array}$ & $\begin{array}{l}\text { Fugel meyer } \\
\text { score FM }\end{array}$ \\
\hline 2 & $\begin{array}{l}\text { Sara } \\
\text { benham, } \\
\text { varleisha } \\
\text { gibbs }\end{array}$ & $\begin{array}{l}\text { Case study } \\
\mathrm{N}=2, \\
\text { Age }=3-12 \\
\text { years }\end{array}$ & $\begin{array}{l}\text { School children } \\
6 \text { weeks, } 20 \\
\text { sessions, } \\
\text { frequency=30 } \\
\text { min. }\end{array}$ & $\begin{array}{l}\text { Telerehabilita } \\
\text { tion motion } \\
\text { capture } \\
\text { programme in } \\
\text { school } \\
\text { settings. }\end{array}$ & $\begin{array}{l}\text { Improved } \\
\text { motor skills } \\
\text { (gross and fine } \\
\text { motor skills) }\end{array}$ & $\begin{array}{l}\text { Bruininks- } \\
\text { oseretsky test } \\
\text { of motor } \\
\text { proficiency } \\
\text { (BOT 2-SF) }\end{array}$ \\
\hline 3 & $\begin{array}{l}\text { Melanie } \\
\text { Joy Criss }\end{array}$ & $\begin{array}{l}\text { Pilot study } \\
\mathrm{N}=8, \\
\text { Age }= \\
6-11 \text { years. }\end{array}$ & $\begin{array}{l}\text { School children, } \\
6 \text { intervention } \\
\text { sessions, } 30 \\
\text { min.session } \\
\text { /week }\end{array}$ & $\begin{array}{l}\text { Virtual } \\
\text { telerehabilitati } \\
\text { on } \\
\text { programme } \\
\text { using web } \\
\text { camera }\end{array}$ & $\begin{array}{l}\text { Improved fine } \\
\text { motor skills } \\
\text { especially } \\
\text { handwriting }\end{array}$ & Print tool $^{\mathrm{TM}}$ \\
\hline
\end{tabular}


Journal of Exercise Science \& Physiotherapy Vol. 14 No. 2 (July to December) 2018

ISSN: 0973-2020 (Print) $\quad \mathrm{I}_{2}$ OR Impact Factor $=6.115$ UGC Approved [no.7485] ISSN: 2454-6089 (Online)

\begin{tabular}{|c|c|c|c|c|c|c|}
\hline 4 & $\begin{array}{l}\text { Amy J. } \\
\text { Brisben } \\
\text {,Charlotte } \\
\text { S. Safos. }\end{array}$ & $\begin{array}{l}\text { Pilot study } \\
\text { N=6, } \\
\text { Age=4-10 } \\
\text { years }\end{array}$ & $\begin{array}{l}\text { Cerebral palsy } \\
\text { children, } \\
16 \text { weeks period, } \\
\text { with once in } \\
\text { week }\end{array}$ & $\begin{array}{l}\text { Robot with } \\
\text { Gestural } \\
\text { sensors }\end{array}$ & $\begin{array}{l}\text { Improved gross } \\
\text { and fine motor } \\
\text { skills, improve } \\
\text { upper } \\
\text { extremity } \\
\text { strength, } \\
\text { coordination } \\
\text { and dexterity }\end{array}$ & Cosmobot $^{\mathrm{TM}}$ \\
\hline 5 & $\begin{array}{l}\text { Diane } \\
\text { Solomon } \\
\text { et al. }\end{array}$ & $\begin{array}{l}\text { Pilot study, } \\
\mathrm{N}=3, \\
\text { Age }=13-15 \\
\text { years }\end{array}$ & $\begin{array}{l}\text { Hemiplegic } \\
\text { Cerebral palsy, } \\
22 \mathrm{hrs}\end{array}$ & $\begin{array}{l}\text { Virtual } \\
\text { rehabilitation } \\
\text { telerehabilitati } \\
\text { on } \\
\text { programme }\end{array}$ & $\begin{array}{l}\text { Improved } \\
\text { gripping } \\
\text { activities }\end{array}$ & $\begin{array}{l}\text { Bruininks- } \\
\text { oseretsky test } \\
\text { of motor } \\
\text { proficiency(B } \\
\text { OT 2-SF) }\end{array}$ \\
\hline 6 & $\begin{array}{l}\text { Pedro } \\
\text { Caro U et } \\
\text { al. }\end{array}$ & $\begin{array}{l}\text { Case study, } \\
\mathrm{N}=3, \\
10-18 \text { years }\end{array}$ & $\begin{array}{lr}\text { Spastic } & \text { Cerebral } \\
\text { palsy, } & \\
10 & \text { sessions } \\
\text { during } & 40 \\
\text { minutes } & \\
& \end{array}$ & $\begin{array}{l}\text { Rehabitic } \AA \\
\text { software }\end{array}$ & $\begin{array}{l}\text { Marked } \\
\text { changes in } \\
\text { strength, tone, } \\
\text { quality of } \\
\text { exercise } \\
\text { performance }\end{array}$ & $\begin{array}{l}\text { GMFCS } 1 \text { and } \\
2\end{array}$ \\
\hline 7 & $\begin{array}{l}\text { Golomb } \\
\text { MR et al. }\end{array}$ & $\begin{array}{l}3 \text { months } \\
\text { Pilot study, } \\
\mathrm{N}=3\end{array}$ & $\begin{array}{l}\text { Hemiplegic } \\
\text { cerebral } \\
\text { palsy,5days/wee } \\
\mathrm{k} \text { for } 30 \mathrm{~min} . \mathrm{a} \\
\text { day }\end{array}$ & $\begin{array}{l}\text { Vrtual reality } \\
\text { videobased } \\
\text { games } \\
\text { rehabilitation } \\
\text { systems using } \\
\text { sensored } \\
\text { glove }\end{array}$ & $\begin{array}{l}\text { Improved } \\
\text { functions of } \\
\text { plegic hand, } \\
\text { improved } \\
\text { finger ROM }\end{array}$ & $\begin{array}{l}\text { Standardized } \\
\text { assessments } \\
\text {,remote } \\
\text { assessments of } \\
\text { finger ROM, } \\
\text { DXA,PQCT, } \\
\text { fMRI of hand } \\
\text { grip task }\end{array}$ \\
\hline 8 & $\begin{array}{l}\text { Danielle } \\
\text { Levac et } \\
\text { al. }\end{array}$ & $\begin{array}{l}\text { pilot non } \\
\text { randomised } \\
\text { control trial } \\
, \mathrm{N}=5\end{array}$ & $\begin{array}{l}\text { Cerebral palsy } \\
\text { ambulatory } \\
\text { children, } \\
7 \text { weeks prog. } \\
1 \text { hr trg. for } 5 \\
\text { days clinical } \\
\text { based } \\
\text { system }\end{array}$ & $\begin{array}{l}\text { Therapist } \\
\text { monitored } \\
\text { videogame } \\
\text { AVG PROG. }\end{array}$ & $\begin{array}{l}\text { Neither } \\
\text { intervention } \\
\text { improved } \\
\text { outcome in } \\
\text { small sample }\end{array}$ & $\begin{array}{l}\text { GMFM-CM, } \\
\text { 6MWT }\end{array}$ \\
\hline 9 & $\begin{array}{l}\text { Judith E } \\
\text { Deutch, } \\
\text { Megan } \\
\text { Borbely, } \\
\text { Jenny } \\
\text { Filler, } \\
\text { Karen } \\
\text { Huhn et } \\
\text { al. }\end{array}$ & $\begin{array}{l}\text { Retrospecti } \\
\text { ve and } \\
\text { prospective } \\
\text { case report } \\
\text { in school } \\
\text { settings ,8- } \\
15 \text { yrs }\end{array}$ & $\begin{array}{l}\text { Cerebral palsy } \\
\text { children, } 11 \text { trg. } \\
\text { sessions of } 60- \\
90 \text { minutes }\end{array}$ & $\begin{array}{l}\text { Wii sport } \\
\text { games } \\
\text {,software }\end{array}$ & $\begin{array}{l}\text { Positive } \\
\text { outcomes with } \\
\text { Wii games }\end{array}$ & $\begin{array}{l}\text { Visual } \\
\text { perceptual } \\
\text { skills test, } \\
\text { functional } \\
\text { mobility and } \\
\text { postural } \\
\text { control } \\
\text { measures }\end{array}$ \\
\hline
\end{tabular}


Journal of Exercise Science \& Physiotherapy Vol. 14 No. 2 (July to December) 2018

ISSN: 0973-2020 (Print) $\quad I_{2}$ OR Impact Factor $=6.115$ UGC Approved [no.7485] ISSN: 2454-6089 (Online)

\begin{tabular}{|l|l|l|l|l|l|l|}
\hline 10. & $\begin{array}{l}\text { Luna } \\
\text { Oliva, } \\
\text { Laura, et } \\
\text { al. }\end{array}$ & $\begin{array}{l}\text { Preliminary } \\
\text { study in } \\
\text { school } \\
\text { environmen } \\
\text { t, N=11 }\end{array}$ & $\begin{array}{l}\text { Cerebral palsy } \\
\text { children, 8 } \\
\text { weeks prog. }\end{array}$ & $\begin{array}{l}\text { Virtual teality tech. } \\
\text { Kinect box } \\
360\end{array}$ & $\begin{array}{l}\text { Improvement } \\
\text { in balance and } \\
\text { ADL skills }\end{array}$ & $\begin{array}{l}\text { GMFM,AMP } \\
\text { S motor, PRT }\end{array}$ \\
\hline 11. & $\begin{array}{l}\text { Anupriya } \\
\text { Kanitkar, } \\
\text { Tony } \\
\text { Szturm, } \\
\text { Sanjay } \\
\text { Parmar et } \\
\text { al. }\end{array}$ & $\begin{array}{l}\text { Randomize } \\
\text { single control } \\
\text { blind, } \\
\text { clinical } \\
\text { trial, } \\
\text { N=140,4 } \\
10 \text { yrs }\end{array}$ & $\begin{array}{l}\text { Cerebral palsy } \\
\text { children, } \\
\text { Control gp.=45 } \\
\text { min./session,3 } \\
\text { times a week for } \\
16 \text { weeks }\end{array}$ & $\begin{array}{l}\text { Computer } \\
\text { based games }\end{array}$ & $\begin{array}{l}\text { Improvement } \\
\text { in gross and } \\
\text { fine motor } \\
\text { skills. }\end{array}$ & $\begin{array}{l}\text { QUEST,PDM } \\
\text { S-2 }\end{array}$ \\
\hline
\end{tabular}

\section{Conclusion}

This mini review study reveals that telephysiotherapy through virtual reality, robotic therapies and other systems of telerehabilitation can develop and promote meaningful functional outcomes in motor skills of children with cerebral palsy as noticed by review of articles. This study suggests that telephysiotherapy for cerebral palsy children is effective mode of treatment in developing motor skills along with educational settings as in schools especially in special schools. Keeping in mind the few articles in study included, telephysiotherapy could be handy mode of therapeutics for children with cerebral palsy in school settings. This paper provides an overview of current literature on telephysiotherapy for cerebral palsy children in improving motor skills in school settings.

\section{References}

Alessandro Peretti, Francesco Amenta, Seyed Khosrow Tayebati (2017). Telerehabilitation: Review of the State-of-the-Art and Areas of Application, JMIR rehabilitation and assistive technologies; 4 (2) 1.

Amy J. Brisben Charlotte S. Safos, Anna D. Lockerd Jack M. Vice, Corinna E. Lathan. The CosmoBot ${ }^{\mathrm{TM}}$ System: Evaluating its Usability in Therapy Sessions with Children Diagnosed with Cerebral Palsy.

Anupriya Kanitkar, Tony Szturm, Sanjay Parmar et al (2017).The effectiveness of a computer game based rehabilitation platform for children with cerebral palsy: protocol for randomized clinical trial. JMIR Res. Protoc; 6(5): e93 doi: 10.2196/ resprot.6846.

Criss M (2013). School-based telerehabilitation in occupational therapy: Using Telerehabilitation technologies to promote improvements in student performance. International Journal of Telerehabilitation, 9(1), 39-46.

Cristinia Bayon, Rafael Rayaz, Sergio Lara (2016). Robotic Therapies for Children with Cerebral Palsy: A Systematic Review. Translational Biomedicine; 7(1:44), 1-10.

Danille Levac, Anna McCormick, Mindy F (2017). Active video gaming for children with cerebral palsy: Does a clinic based virtual reality component offer an additive benefit? A pilot study. Physical \& occupational therapy in paediatrics; 38(1).

Diane solomen, Pam Bernard (2010). Efficacy of active video game to improve outcomes for children with cerebral palsy- fact sheet, TRIPSKY- Evidence based journal club;5(1), 1-2.

Gilberto Marzano and Velta Lubkina (2016). A review of Telerehabilitation Solutions for Balance Disorders. Procedia Computer Science 104, 250 - 257.

Golomb MR, Mc Donald BC, Warden SJ et al (2010). In home virtual reality videogame telerehabilitation in adolescents with hemiplegic cerebral palsy. Arch Physical Med Rehabil; 91:1-9.

Jana Cason (2011). Telerehabilitation: An Adjunct Service Delivery Model for Early Intervention Services, International Journal of Telerehabilitation; 3(1).

Josip Glavic, Stela Rutović, Nikolina Kristić-Cvitanović, Petra Burić (2016).Technology- Enhanced Upper Limb Physical Rehabilitation in Hemiplegic Cerebral Palsy. Int J Neurorehabilitation Eng; 3:4, 1-2. 


\section{Journal of Exercise Science \& Physiotherapy Vol. 14 No. 2 (July to December) 2018 \\ ISSN: 0973-2020 (Print) I2OR Impact Factor =6.115 UGC Approved [no.7485] ISSN: 2454-6089 (Online)}

Judith E Deutch, Megan Borbely, Jenny Filler, Karen Huhn et al (2008). Use of low cost, commercially available gaming console (Wii) for Rehabilitation of an adolescent with cerebral palsy. Physical therapy; 88 (10) 1196 - 1207.

Luna Oliva L, Ortiz-Gutierrez RM, Cano-de la Cuerda R (2013). Kinect Xbox 360 as a therapeutic modality for school children with cerebral palsy in school environment: A preliminary study. Neuro Rehabilitation; 33(4): 513-521.

Sara benham, Varleisha gibbs (2017). Exploration of the effects of telerehabilitation in a school-based setting for at-risk youth. International Journal of Telerehabilitation; 9 (1), 39-46.

Thais Massetti, Talita Dias da Silva, Denise Cardoso Ribeiro (2014). Motor learning through virtual reality in cerebral palsy. Medical express; 1(6):302-306.

Young TL. Ireson C. (2003).Effectiveness of school-based telehealth care in urban and rural elementary schools. Paediatrics; 112:1088-1094.

Conflict of Interest: None declared 